\title{
The Education Value Of Cloud Computing
}

\author{
Harry Katzan, Jr., Savannah State University, USA
}

\begin{abstract}
Cloud computing is a technique for supplying computer facilities and providing access to software via the Internet. Cloud computing represents a contextual shift in how computers are provisioned and accessed. One of the defining characteristics of cloud software service is the transfer of control from the client domain to the service provider. Another is that the client benefits from economy of scale on the part of the provider. Cloud computing is particularly attractive to small and medium-sized educational institutions, because it represents a lower total cost of ownership (TCO) than alternative modalities.
\end{abstract}

Keywords: Cloud computing, software-as-a-service, platform-as-a-service, infrastructure-as-a-service, private cloud, public cloud, community cloud, hybrid cloud.

\section{INTRODUCTION}

$Q$ s modern education, government, and business, have evolved into the 21 st century, the use of computers to sustain everyday operations has increased. Most organizations employ computers to enhance core services and provide supplementary services to gain efficacy and efficiency for auxiliary operations. One of the newest technologies for IT provisioning is cloud computing that has garnered a considerable amount of attention in the business, education, and government communities. Many persons in small business, universities, and schools are not totally aware of the benefits of cloud computing, and that is the reason for this paper. First, we will cover what cloud computing is; then we will cover how it works; next we will cover how to get it; and finally, we will provide the pros and cons of adopting it as your mode of operation.

Cloud computing is a means of providing computer facilities via the Internet, but that is only half of the picture. The other half is that it is also a means of accessing those same computer facilities via the Internet from different locations. When a school or university, for example, moves to cloud computing for online operations, it necessarily considers both halves of the equation. The adjective "cloud" reflects the diagrammatic use of a cloud as a metaphor for the Internet. In telecommunications, a cloud is the unpredictable part of a network through which organizational and personal information passes from end-to-end and over which we do not have direct knowledge or control. ${ }^{1}$

The value proposition that underlies cloud computing is that an organization does not have to pay the upfront costs of hardware, software, networks, people, training, and other infrastructural elements. Instead, the organization would utilize resources provided on the Internet as they would a service utility, such as electric service and pay only for what it uses. The service would take care of peak periods, support, downtime, training, and a myriad of other things with which an organization would possibly prefer not to get involved. Another important consideration is that many organizations that depend on online services have to plan for a worst-case scenario and experience low server utilization even during peak periods. Some major banks have a server use percentage as low as $6 \%$ during normal periods and $20 \%$ during peak periods.

The essence of cloud computing is service. The company providing cloud computing service assumes the role of service provider, and the organization using the service takes on the role of the client (or customer). Services are indigenous to the existence of modern society and are constantly being invented and retired. So we should be right at home with cloud computing. At least, that is what major computer and software companies, major universi-

\footnotetext{
${ }^{1}$ See Privacy in the Clouds by Cavoukin for more information on this subject.
} 
ties, the U.S. Government, and the National Institute of Standards and Technology (NIST) seem to think. ${ }^{2}$

\section{CHARACTERISTICS OF CLOUD COMPUTING}

Computing is a social phenomena based on technology, the basis of which is input, processing, output, and storage. If the task is an ordinary computer application, such as word processing, payroll, use of the cell phone, or checkout at the supermarket, there is always the aforementioned four steps known as the information processing cycle. In a very general sense, input can come from people and machines, processing is done by computers, output goes to people or machines, and information produced or absorbed during computation can be stored for future use on electronic devices. Where in an organizational sense each of the steps actually takes place determines if it is cloud computing or traditional "on premises" computing. Relying on evolutionary processes, we can observe that the structure of an organism essentially determines its potential for growth. So it is with cloud computing.

A cloud computing service would necessarily have ubiquitous access through a Web browser or mobile device providing the input step. The computing and storage facilities would reside in and operate from a data center in the cloud. The output is returned to the end user through the browser program or mobile device, mentioned previously. In order to sustain the cloud operational environment, a cloud computing service would provide a utilitylevel infrastructure with the following operational characteristics: necessity, reliability, usability, and scalability. Necessity refers to the idea that a preponderance of users depend on the service to satisfy everyday needs. Reliability refers to the expectation that the service will be available when the user requires it. Usability refers to the requirement that the service is easy and convenient to use - regardless of the complexity of the underlying infrastructure. Scalability refers to the fact that the service has sufficient capacity to allow the users to experience the benefits of an expandable service that provides economy of scale. Certainly, modern Internet facilities for search operations that typically engage thousands of servers satisfy these characteristics.

\section{APPLICATION SERVICE PROVISIONING}

The need for effective computer service provisioning has been on the sidelines for some time and represents a unrealized requirement in the education, government, and business worlds. The situation is that small to medium-sized organizations have need for expensive computing facilities and software service. A prototypical example is the small software firm that needs occasional mainframe computer time. The solution has been to lease service from an application service provider (ASP) and use that service via network facilities. The ASP supplies the computer time and provides operational software as required. The process is known as hosting. The customer assumes the network expenses without the up-front hardware, software, and facilities costs.

With cloud computing, the Internet provides the network facilities. A cloud service provider supplies the computer and operating system resources that can be accessed via the Internet. Applications software is supplied by an independent software vendor (ISV) and therein resides the benefit to the customer. The customer, which can have several users, shares the software among several customers so as to achieve significant economy-of-scale.

\section{EDUCATION AND CONSUMER SERVICE}

Chong and Carraro at Microsoft ${ }^{3}$ define shared software as software-as-a-service (SaaS) deployed as a hosted service and accessed over the Internet. The key features of SaaS are where the programs reside and how they are accessed. The two kinds of software in this category are education software and consumer software. Education software provides education services and emphasizes education solutions, such as registration, educational accounting, record keeping, online learning, and human resources. Cloud computing is an ideal means of managing district computers. Consumer software provides personal solutions, such as office applications, that are often available at no cost in their cloud versions.

\footnotetext{
${ }^{2}$ See the NIST definition of cloud computing by Mell and Grance.

${ }^{3}$ See Chong, 2006.
} 
With education services, the most important consideration is whether the programs are executed in-house or as a cloud service. When the process is handled in-house, total control over the operation is obtained along with limited opportunity for achieving economy-of-scale. As processes are distributed outward on the cloud, control is decreased but opportunities for achieving economy-of-scale are increased. The considerations are different with consumer services. Pure service, as with office applications, provides practically no control over the application to the client and a reasonably high-level of economy-of-scale to the provider. In many cases, consumer services are advertising-supported and are complimentary to the client through advertising. In addition to metered and subscription models, the advertising-supported model is another means of monetizing cloud computing.

Education applications that reside "on premises" are governed by the traditional considerations of application acquisition and deployment. If an application resides on and is deployed from the cloud, then two options exist:

(1) Build the software yourself (or have it built for you) and run it on the cloud as a hosted service - perhaps using a cloud platform.

(2) Obtain the application software from an independent software vendor (ISV) and run it on the cloud in a standard or modified mode.

In the former case, all users access the same version of the software. In the latter case, a client gets a customized version achieved with a separate code base (or its equivalent) and configuration options. A note on terminology is in order, especially with regard to traditional software. In a prototypical cloud environment, there are multiple service entities providing service, such as the cloud infrastructure provider (i.e., the computer part) and the ISV (i.e., the software part).

The two entities are combined for discussion purposes into a single service provider that we are going to conceptually refer to as the cloud software service (CSS). Two institutions, for example, contract with the CSS for access to and the execution of education applications in areas such as record-keeping, scheduling, infrastructure management, and so forth. The companies are referred to as customers of the CSS. Each customer entrusts several employees to use the contracted services, and they are regarded as the users. In some instances, the customers and their respective users are considered to be clients of the service provider.

The primary advantage of a cloud consumer service is that it is typically free to the client, as well as being accessible from any location via the Internet, and it yields advertising-supported revenue for the provider. Consumer services have a near-zero marginal cost of distribution to clients, requiring only a fraction of the number of clients to respond to advertising. This is the well-known Freemium Business Model ${ }^{4}$, characterized as follows: In the free sample product model, you give away $1 \%$ of your product to sell the additional $99 \%$, whereas in the freemium model, you give away $99 \%$ to sell $1 \% .^{5}$ Because of the scale of the Internet with millions of users, you can reach a large market, so that the $1 \%$ is a huge amount.

Clearly, the conceptual model for the deployment of SaaS changes with the adoption of cloud computing. The ownership of software shifts from the client to the provider, along with the responsibility for the technology infrastructure and its management ${ }^{6}$.

\section{CLOUD PLATFORMS AND SERVICE DEPLOYMENT MODELS}

A cloud platform is an operating system that runs in the cloud and supports the software-as-a service concept. A cloud platform resides in a cloud data center and exists as a powerful computing facility, a storage system, an advanced operating system, support software, and the necessary fabric to sustain a server farm and scale up to support millions of Internet clients. A cloud platform is as much about operating in the cloud, as it is about developing applications for the cloud. A cloud platform provides the facility for an application developer to create applications that run in the cloud; and, in so doing, the application developer uses services that are available from the cloud.

\footnotetext{
${ }^{4}$ See Anderson 2006.

${ }^{5}$ The percentages should not be taken literally, in this instance. They are used only to make a point (Anderson 2006).

${ }^{6}$ See Chong 2006.
} 
Cloud platforms are a lot like enterprise-level platforms, except that they are designed to scale up to Internet-level operations supporting millions of clients.

The essential elements of a cloud service deployment are given above. In order to develop enterprise-wide applications, a comprehensive viewpoint has to be assumed with deployment models from the following list: (Mel op cit.)

Private cloud. The cloud infrastructure is operated solely for an organization. It may be managed by the organization or a third party and may exist on premise or off-premise.

Community cloud. The cloud infrastructure is shared by several organizations and supports a specific community that has shared concerns (e.g., mission, security requirements, policy, and compliance considerations). It may be managed by the organizations or a third party and may exist on-premises or off-premises.

Public cloud. The cloud infrastructure is made available to the general public or a large industry group and is owned by an organization selling cloud services.

Hybrid cloud. The cloud infrastructure is a composition of two or more clouds (private, community, or public) that remain unique entities but are bound together by standardized or proprietary technology that enables data and application portability (e.g., cloud bursting for load-balancing between clouds).

Many cloud software service application domains will be synthesized from a combination of the deployment models.

\section{CLOUD SERVICE ECONOMICS}

Cloud services mark a milestone in IT service provisioning. The cloud model promotes availability and operates through a large ecosystem of different approaches to on-demand accessibility supplied by vendors and various market niches. The pool of shared resources essentially determines the economics of the cloud paradigm.

Cloud service democratization refers to either of three distinct but related forms. In the first instance, known as the availability model, it is the process of making a premium cloud service available for general use, rather than through proprietary services. In the second instance, known as the sharing model, it is the capability of sharing data, infrastructure, and storage that would not be otherwise accessible with on-premises facilities. The final instance, known as the voting model, is the phenomena of giving power to the end user by providing access to facilities that are implicitly more preferable than other cloud resources by virtue of the fact that they are used or referenced more frequently by other end users.

The availability model reflects the ability of having access to information, software, and computing resource infrastructure without necessarily having to own them. In many cases, the cost and time elements are too high for many organizations, because the up-front costs and time to develop the information, software, and onpremises resources is simply too great for many potential clients and ISVs. The cost of providing computing infrastructure and software by traditional ISVs is such that it is affordable only by larger institutions. This situation leaves out the long tail ${ }^{7}$ of small to medium-sized organizations that could benefit from the solution, if the cost were lower. By lowering the cost of service provisioning by utilizing multi-tenancy technology and taking advantage of economy of scale achieved through multiple clients from the cloud, education software services are available to the long-tail market.

\footnotetext{
${ }^{7}$ The long tail (Cho06) is a conceptualization of the unique business opportunities available through Internet access, exemplified by online book sellers and software services. A brick-and-mortar bookseller has a limited amount of self space and typically stocks only the most popular books. Online booksellers do not have the same limitation and are able to take advantage of lower inventory costs, known as the "long tail." The long-tail phenomenon also applies to application software and consumer-oriented services.
} 
The sharing model refers to the fact that three major classes of technical resources are available, on a shared basis, through the cloud platforms: data and information, infrastructure services, and data storage facilities. In the case of data and information, content can be shared between users from the same client, between clients, and between platforms from the same user. Effectively, more information is available to more users. Infrastructure sharing is a major category of cloud service and is a major cost to an IT shop. It includes software, hardware, and security services. With software, comprehensive facilities are available at a lower price, because the cost is shared among thousands of users. With hardware, the end user does not need to plan for peak periods and growth, since elasticity is designed into the architecture of cloud platforms. This is the "scalability" characteristic of utility services. For security, federated security systems are shared among users and clients enabling mobility between diverse computing platforms. Data storage sharing refers to the common habitation of data on a cloud platform by several clients. Storage multiplicity, commonly available on cloud platforms, reduces organizational concerns in the general area of disaster planning.

The value of certain services, such as web auctions (e.g., e-Bay), user-supported encyclopedias (e.g., Wiki), and modern search engines (e.g., Google), is derived from the fact that many people use them. This is known as the voting model. The power of such facilities lies in the fact that each individual user votes by choosing to use the respective service. With a web auction, it is the interchange between users that gives the facility its democratic power. With an updatable online encyclopedia, an individual end user has the power of updating an entry. This option allows the informational resource to evolve as more people use it. With a search engine, such as Google, it is the method of page ranking, wherein the number of page references to an object page gives its score, and allows a universe of users to democratize a page. Collectively, cloud service democratization essentially enables the delivery of computing service to more clients at a reduced cost.

The basis for the monetization of cloud computing is software-as-a-service (SaaS), commonly regarded as software hosted service from a cloud platform. The varieties of software-as-a-service are single-tenant, and multitenant. With single-tenant SaaS, the service provider delivers a unique set of features that are hosted in the cloud through a cloud platform and Internet accessibility to the client. The client has isolated access to a common set of features, perhaps configured in a distinctive way. With multi-tenant SaaS, the provider hosts common program logic and unique data elements for multiple clients on scalable infrastructure resources supported via a cloud platform. With multi-tenant, the clients share the operation of the software.

\section{CLOUD SERVICE MODELS}

A set of cloud service models give an ontological view of what a cloud service is. A cloud service system is a set of elements that facilitate the development of cloud applications. Here is a description of the three layers in the NIST service model description: (Mel op cit.)

Cloud Software as a Service (SaaS). The capability provided to the consumer is the use of the provider's applications running on a cloud infrastructure. The applications are accessible from various client devices through a thin client interface such as a web browser (e.g., web-based email). The consumer does not manage or control the underlying cloud infrastructure including network, servers, operating systems, storage, or even individual application capabilities, with the possible exception of limited user-specific application configuration settings.

Cloud Platform as a Service (PaaS). The capability provided to the consumer is that of deploying onto the cloud infrastructure consumer-created or acquired applications developed through the use of programming languages and tools supported by the provider. The consumer does not manage or control the underlying cloud infrastructure including network, servers, operating systems, or storage, but has control over the deployed applications and possibly application hosting environment configurations.

Cloud Infrastructure as a Service (IaaS). The capability provided to the consumer is the capability of provisioning processing, storage, networks, and other fundamental computing resources where the consumer is able to deploy and run arbitrary software, which can include operating systems and applications. The consumer does not manage or control the underlying cloud infrastructure but has control over the operating system, storage, and deployed applications, as well as limited control over selected networking components (e.g., host firewalls). 
The three service model elements should be deployed in a cloud environment with the essential characteristics in order to achieve a cloud status

\section{QUICK SUMMARY}

1. Cloud computing is a means of accessing computer facilities via the Internet. (The cloud is a metaphor for the Internet.)

2. Cloud service facilities are characterized by four key factors: necessity, reliability, usability, and scalability.

3. Software-as-a-service (SaaS) is software deployed as a hosted service and accessed over the Internet.

4. For the cloud client, education service is a balance between control and economy of scale.

5. A cloud platform is based on an operating system that runs in the cloud and provides an infrastructure for software development and deployment.

6. Cloud service democratization refers to information and computing availability, information sharing, and the exercise of user preference in supplying information service.

7. Cloud service monetization refers to gaining financial benefit through cloud access and economy-of-scale for both provider and client.

\section{ACKNOWLEDGMENT}

Thanks to Margaret Katzan for reading the manuscript.

\section{AUTHOR INFORMATION}

Dr. Harry Katzan teaches at Savannah State University and is the founding editor of the Journal of Service Science.

\section{REFERENCES AND SELECTED READING}

1. Anderson, C. 2006. The Long Tail. New York: Hyperion.

2. Cloud Computing: The Evolution of Software-as-a-Science. 2008. Arizona State University W.P. Carey School of Business, June 4, 2008, knowledge.wpcarey.asu.edu.

3. Cavoukian, A. 2009. Privacy in the Clouds. Toronto: Information and Privacy Commission of Ontario (www.ipc.on.ca).

4. Chappell, D. 2008 A Short Introduction to Cloud Platforms. Microsoft Corporation.

5. Chong, F. and G. Carraro. 2006. Architecture Strategies for Catching the Long Tail. Microsoft Corporation.

6. Chong, F. 2008. Application Marketplaces and the Money Trail. Microsoft Corporation.

7. IBM Corporation. 2009. The Benefits of Cloud Computing. Form DW03004-USEN-00.

8. Katzan, H. 2009. Cloud Software Service: Concepts, Technology, Economics. Service Science, 1(4):256269.

9. Martin, R and J. Hoover. 2008. Guide to Cloud Computing. Information Week, June 21, 2008, www.informationweek.com.

10. Mell, P. and T. Grance. 2009a. The NIST Definition of Cloud Computing. National Institute of Standards and Technology, Information Technology Laboratory, Version 15, 10-7-09. (http://www.csrc.nist.gov/groups/SNS/cloud-computing/index.html)

11. Mell, P., Badger, L., and T. Grance. 2009b. Effectively and Securely Using the Cloud Computing Paradigm. National Institute of Standards and Technology, Information Technology Laboratory, 10-7-09. (http://www.csrc.nist.gov/groups/SNS/cloud-computing/index.html)

12. Miller, M. 2008. Cloud Computing: Web-Based Applications That Change the Way You Work and Collaborate Online, Indianapolis: Que Publishing.

13. Rappa, M. 2004. The utility business model and the future of computing services. IBM Systems Journal, 43(1):32-41.

14. Reese, G. 2009. Cloud Application Architectures: Building Applications and Infrastructure in the Cloud, Sebastopol, CA: O'Reilly Media, Inc. 